\title{
Predicting crack resistance by infrared thermography
}

\author{
by G. Kurilenko
}

Novosibirsk State Technical University, K. Marx Str., 20, 630092, Novosibirsk, Russia

\begin{abstract}
For the last years, the problem of predicting crack resistance found increasing interest. This could be explained by insufficient accuracy of traditional techniques. The proposed method is based on the information coming from kinetics of temperature distributions appearing on the surface of tested specimens. As the method involves the use of IR thermographic equipment, it offers some advantages compared with traditional techniques.
\end{abstract}

\section{Introduction}

It is well known that cyclic deforming of materials is accompanied by generation of heat. Thermal phenomena could be considerable even under macroelastic deformation. If, in addition, the amplitude of stress exceeds the endurance limit, microplastic deformations of separate unfavourably-oriented crystals of metal will occur. In their turn, these deformations will become local heat sources. Therefore, processes of macroelastic loading have to be considered together with the thermodynamics of microplastic deformations. As a result, specific temperature distributions which characterize irreversible material changes will appear. These distributions supply information about the damage process.

Our experiments performed with main types of steel have shown that the problem of accuracy in predicting crack resistance can be solved to a certain extent by using the thermographic method.

Within the method used, we have applied such criterions of damage as $q_{v}$ - the intensity of heat source in the domain of damage and $\Delta S_{1 \mathrm{c}}$ - the change of specific entropy for one cycle of an oscillation correlated only to energy dispersion in the form of heat, and calculated with the formula:

$$
\Delta S_{1 c}=c_{v} \ln \left(T_{2} / T_{1}\right) .
$$

Here $c_{v}$ is the specific heat capacity of the material; $T_{2}$ and $T_{1}$ are the temperatures on the surface of the domain at the end and beginning of a particular cycle.

\section{Experimental definition of the intensity of heat source in the domain of damage [1]}

The first experiments were performed on thinwalled pipe-type specimens which contained symmetrically located stress concentrators. The oscillations were induced along their length. The cyclic loading of specimens has been made step by step with increasing amplitude of stress $\sigma_{a}$ starting from stresses below the endurance limit to stresses above it. At the end of every step of loading the heat field became relatively stabilized (beginning of the second stage) and we determined the temperature curves along the surface (figure 1). Their shape is strongly influenced by the heat source generated by stress concentration in the middle of the sample. Its intensity $q_{v}$ is an important parameter because through it the cyclic lifetime of testing object can be defined.

To calculate $q_{v}$ at each step, the corresponding experimental temperature curves (figure 1) have been analysed.

As the regression equation we used the equation of thermal conduction where also convective heat exchange is considered:

$$
\rho c_{v} \frac{\partial T}{\partial t}=\lambda \frac{\partial^{2} T}{\partial x^{2}}+q-r\left(T-T_{0}\right)
$$


Here $q$ is the specific heat output of energy in the regular part of a sample; $r=2 \pi \alpha{ }_{k} R / A$; $\alpha_{k}$ - coeffiecient of conventional heat's retum; $2 R$ - external diameter of sample. A - the area of the sample cross section; the other definitions are trivial. The parameter $q_{v}$ is introduced in equation (2) through the boundary condition (figure 1):

$$
\left.\frac{\partial T}{\partial x}\right|_{\xi=0}=-q_{v} / 2 \lambda A
$$

Taking into account that the temperature at the end of each step is quasi-stabilized, equation (2) could be simplified. It was assumed that $\frac{\partial T}{\partial t}=0$.

Finally, under the last condition, we have solved equation (2) and obtained the regression function as follows:

$$
T=T_{2} X+\left(T_{0}+q / r\right)(1-X)+q_{v}[X \exp (f L)-\exp (f x)] /\left(2 A f_{1}\right)
$$

Here $T_{2}$ is the temperature at the ends of sample; $X=\operatorname{ch}(f x) / \operatorname{ch}(f L) ; \quad f=(r / \lambda)^{0,5}$; $f_{1}=(r \lambda)^{0,5}$.

On the basis of equation (4) we have treated experimental temperature curves taking temperature values in five points. As a result, the $q_{v}$ values were calculated at each step of loading.

To evaluate the accuracy of the proposed method, we computed theoretical temperature curves using equation (4) and compared them to experimental data. The discrepancy was rather small.

We also calculated $q_{v}$ values using the traditional method (equations of energy balance). The comparison of results has shown that the thermographic method has some advantages in accuracy.

\section{Determination of individual endurance limits [1 - 5]}

Kinetics of an entropy flux $\Delta S_{1 c}$ depending on stress amplitude $\sigma_{a}$ in the domain of damage has been studied with samples of various shapes made of steel 20,30, 45 and 50 under different stepped loads (stretching - compressing, torsion, bending). Testing loads were the same as described before.

We found that $\Delta S_{1 c}-\sigma_{a}$ curves have sharp bends close to a certain threshold stress: the entropy increase $\Delta S_{1 c}$ during the cycle with a small $\sigma_{a}$ value is not significant, but after having overcome this threshold, the stress increase $\Delta S_{1 c}$ is much stronger.

This conclusion fits to modern ideas on the genesis and accumulation of fatigue damage, supposing that these processes accelerate if stresses are above the endurance limit $\sigma_{R}$.

These bends could be seen in the graphics of figure 2 for samples made of steel $\mathbf{4 5}$ under bending loads.

Both statistical data evaluation and comparison with corresponding results obtained with other methods (e.g. with the method of internal friction proposed by us earlier for determination of endurance limits) have shown that these threshold stresses could be taken as individual endurance limits for tested objects.

It should be emphasized that the determination of $\Delta S_{1 \mathrm{c}}$ could be performed by direct recording of the temperature on the surface of a specimen without calculation of some additional parameters. This factor enhances significantly the accuracy and reduces the time of endurance limit prediction in comparison with traditional methods. 


\section{Prediction of crack cyclic resistance $[4,6,7]$}

Traditionally, in fracture mechanics this problem is solved on the basis of the Paris formula (or its modifications) which connects the velocity of crack growth (SCG) to the coefficient of stress intensity (CSI). However, the CSI parameter is not identical to the SCG because CSI does not consider such processes as creation of a plastic zone at the top of a crack, possible branch of cracks under its development etc. That is why prediction of a specimen cyclic lifetime using the Paris formula produces considerable errors.

In order to overcome this problem, we have proposed to use another diagnostic parameter, particularly the $\Delta S_{1 \mathrm{c}}$ value but not the CSI.

To predict cyclic lifetime for a specimen with initial macrocracks, the following empiric dependence has been used:

$$
\frac{d l}{d n}=v^{*}\left[\Delta S_{1 c}(n) / \Delta S_{1 c}^{*}\right]^{m}
$$

which can be considered as a particular kinetic fatigue diagram made using inspection results for samples with their further destruction (figure 3).

In the last expression, the $\frac{d l}{d n}$ member is the SCG; $I$ is the length of the crack; $n$ is the number of loading cycles; $\Delta S_{1 c}{ }^{*}$ is the increment of specific entropy for one cycle at the top of the crack when the SCG is given $\left(v^{*}\right) ; m$ is the empiric coefficient.

It should be mentioned that the parameter $q_{v}$ could be used instead of the diagnostic parameter $\Delta S_{1 c}$.

Eq. (5) must be integrated to the value of resource of samples under a constant regime of cyclic loading:

$$
\Delta l_{c r}=v^{*} \int_{0}^{\mathrm{g}_{c}}\left[\frac{\Delta \mathrm{S}_{\mathrm{lc}}(\mathrm{n})}{\Delta \mathrm{S}_{\mathrm{lc}}^{*}}\right]^{\mathrm{m}} \mathrm{dn} .
$$

The last formula determines cyclic lifetime $n_{\mathrm{or}}$ of the specimen according to crack growth by its critical length $I_{\text {cr. }}$.

The functional dependence $\Delta S_{1 c}(n)$ could be obtained both with a test bench and on the basis of measuring the temperature during industrial exploitation of a component. Otherwise, some results could be obtained simultaneously with making a kinetic fatigue diagram $\frac{d l}{d n}=f\left[\Delta S_{1 c}(n)\right]$. In this case, it can be regarded as the parameter of material.

As a rule, this function could be presented as

$$
\Delta S_{1 c}(n)=\beta n^{\alpha} .
$$

As stated above, here $\alpha$ and $\beta$ are empirical coefficients (parameters of a tested specimen or material).

The comparison of calculated and true lifetimes of tested objects has shown that the accuracy in predicting cyclic resource is much higher than using traditional approaches, both for one - step and mult - step loading. Besides this, the thermographic approach allows to broaden the variety of parts to be inspected.

In our opinion, this means that the $\Delta S_{1 c}(n)$ parameter is more universal and convenient than the CSI parameter in the traditional Paris formula due to the fact that diagnostic criteria $\Delta S_{10}(n)$ or $q_{v}(n)$ allow to take into account individual peculiarities of tested objects. 


\section{Conclusion}

The proposed quantitative thermographic method of predicting crack resistance is based on the use of new thermodynamic criteria of damage development, i.e. increment of entropy for one cycle of an oscillation $\Delta S_{1 c}$ or heat source intensity in the domain of damage $q_{\mathrm{v}}$. These criteria are easily handled through kinetics of temperature distributions which arise during the tests of a specimen.

The method described has some advantages in comparison with traditional approaches. These advantages come from its higher accuracy, efficiency and universal applicability.

The practical implementation of the proposed thermographic method could be made using up-to-date infrared equipment.

This method is successfully used in the inspection of cyclic durability of real parts at some industrial enterprises in Russia.

\section{REFERENCES}

[1] KURILENKO (G.A.). - The usage of thermodynamic approach at cyclic testing of material. PMTF, $n^{\circ} 5,1989$, p. $158-163$.

[2] KURILENKO (G.A.). - Thermodynamic evaluation of the fatigue indurance limit. Plant Laboratory, $\mathrm{n}^{\circ}$ 2, 1989, p. 82 - 85 (in Russian).

[3] The USSR patent No. 1499167. - The method of determining the endurance limit. G.A. Kurilenko, Discoveries. Inventions, $n^{\circ} 29,1989$, p. 169 (in Russian).

[4] KURILENKO (G.A.). - Advanced infrared examing of the metals fatigue. Proc. of the Workshop "Advanced infrared technology and applications", Capri, 1995, p. $253-262$.

[5] KURILENKO (G.A.). - Evaluation of parts cyclic durability through entropy changing in the fatigue domain. Sov. J. NDT (Defectoscopy), $n^{\circ} 7,1989$, p. $53-57$.

[6] KURILENKO (G.A.) and PSHENICHNY (A.B.). - The investigation of metals damage through thermal field kinetics. Proc. of the QIRT'92 Eurotherm Seminar No.27 "Quantitative Infrared Thermography", Paris, 1992, p. $145-149$.

[7] KURILENKO (G.A.), PSHENICHNY (A.B.) and TRUFANOVA (T.V.) - The evaluation of damaging of cyclic deforming parts with macrocracks. TD and NC, $n^{\circ} 3,1992$, p. $46-49$. 
http://dx.doi.org/10.21611/qirt.1996.015

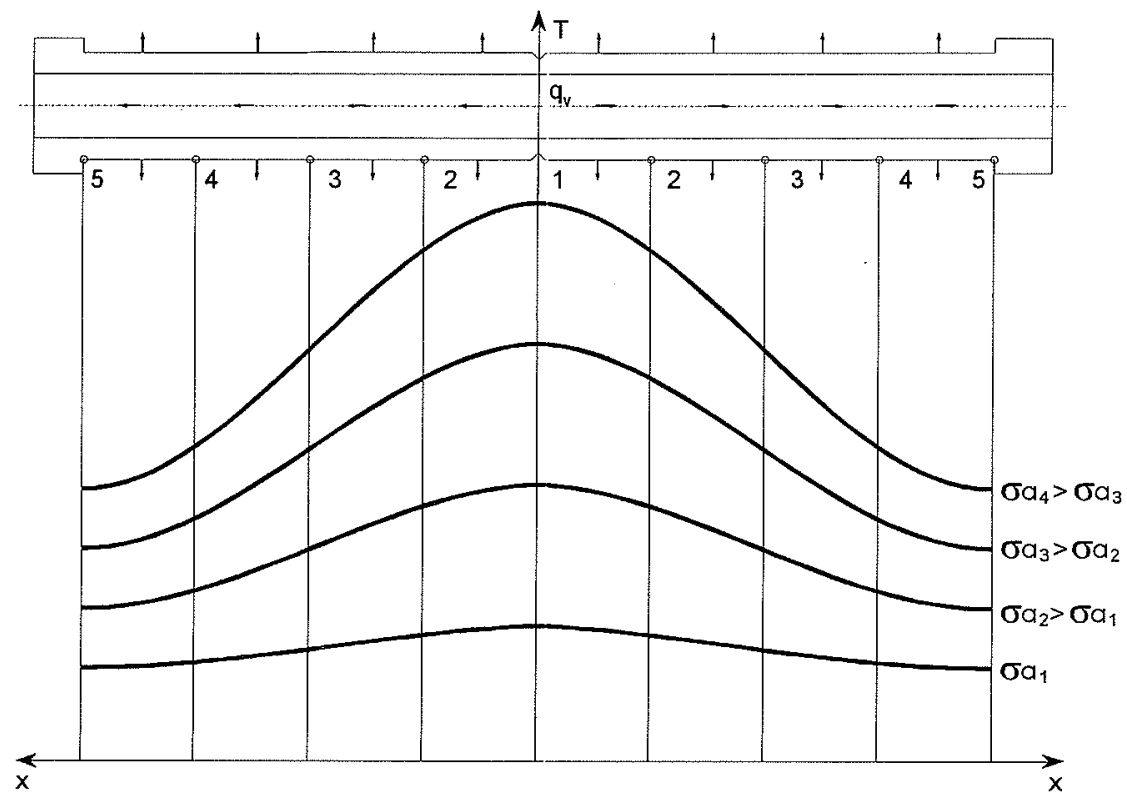

Fig. 1. The temperature kinetics on the surface of sample

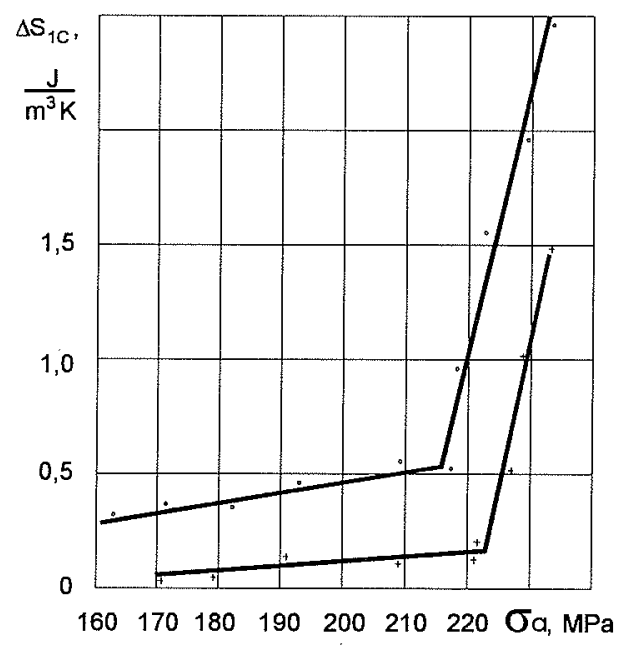

Fig. 2. Empiric dependences received for prediction endurance limits of details

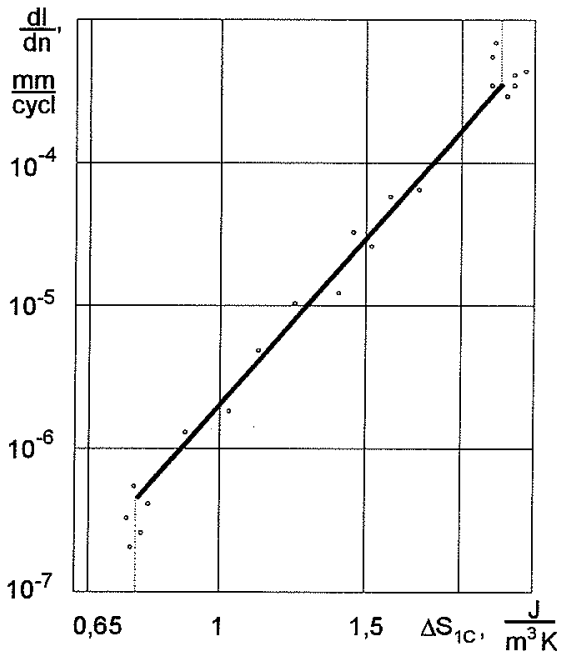

Fig. 3. Thermodynamic kinetic fatigue diagram, built for steel 20 